\title{
Creeping motions of a porous spherical shell in a concentric spherical cavity
}

\author{
H.J. Keh*, Y.S. Lu \\ Department of Chemical Engineering, National Taiwan University, Taipei 106-17, Taiwan, ROC
}

Received 16 September 2004; accepted 19 March 2005

\begin{abstract}
The quasisteady translation and steady rotation of a spherically symmetric porous shell located at the center of a spherical cavity filled with an incompressible Newtonian fluid are investigated analytically. In the fluid-permeable porous shell, idealized hydrodynamic frictional segments are assumed to distribute uniformly. In the limit of small Reynolds number, the Stokes and Brinkman equations are solved for the flow field of the system, and the hydrodynamic drag force and torque exerted by the fluid on the porous shell which are proportional to the translational and angular velocities, respectively, are obtained in closed form. For a given geometry, the normalized wall-corrected translational and rotational mobilities of the porous shell decrease monotonically with a decrease in its permeability. The boundary effects of the cavity wall on the creeping motions of a porous shell can be quite significant in appropriate situations. In the limiting cases, the analytical solutions describing the drag force and torque or mobilities for a porous spherical shell in the cavity reduce to those for a solid sphere and for a porous sphere. The hydrodynamic behavior for a porous spherical shell may be approximated by that for a permeable sphere when the porous shell is sufficiently thick, depending on its permeability.
\end{abstract}

(C) 2005 Elsevier Ltd. All rights reserved.

Keywords: Porous spherical shell; Multiphase flow; Creeping flow; Boundary effects

\section{Introduction}

The motion of colloidal particles in a continuous medium at low Reynolds numbers has long been an important subject in the fields of chemical, biomedical, and environmental engineering and science. The majority of these transport phenomena are fundamental in nature, but permit one to develop rational understanding of many practical systems and industrial processes such as sedimentation, flotation, electrophoresis, agglomeration, and motion of blood cells in an artery or vein. The theoretical study of this subject has grown out of the classic work of Stokes' (1851) for the creeping translational motion of a rigid sphere in an unbounded incompressible Newtonian fluid.

In most practical applications, particles are not isolated. So, it is important to determine if the presence of neighboring particles and/or boundaries significantly affects the movement of particles. Problems of the hydrodynamic interactions between two or more solid or fluid particles and between these particles and various boundaries have been

\footnotetext{
*Corresponding author. Tel.: + 886233663048 ; fax: + 886223623040.

E-mail address: huan@ntu.edu.tw (H.J. Keh).
} 


\begin{tabular}{|c|c|}
\hline Nomenclature & hydrodynamic torque exerted on the porous \\
\hline $\begin{array}{l}\text { internal radius of the porous spherical shell, } \\
\mathrm{m}\end{array}$ & $\begin{array}{l}\text { hydrodynamic torque exerted on the porous } \\
\text { shell in the absence of the cavity, } \mathrm{Nm}\end{array}$ \\
\hline $\begin{array}{l}\text { external radius of the porous spherical shell, } \\
\mathrm{m}\end{array}$ & $\begin{array}{l}\text { translational velocity of the porous shell, } \\
\mathrm{m} \mathrm{s}^{-1}\end{array}$ \\
\hline radius of the spherical cavity, $m$ & fluid velocity field, $\mathrm{m} \mathrm{s}^{-1}$ \\
\hline $\begin{array}{c}A, B, C_{1}, D_{1} \text { coefficients in Eq. (12a) for the external } \\
\text { flow field, given by Eqs. (A.11)-(A.14) }\end{array}$ & $\begin{array}{c}v_{r}, v_{\theta}, v_{\phi} \text { components of fluid velocity in spherical } \\
\text { coordinates, } \mathrm{m} \mathrm{s}^{-1}\end{array}$ \\
\hline $\begin{array}{cl}A^{\prime}, B_{1}^{\prime} & \text { coefficients in Eq. (27a) for the external flow } \\
& \text { field, given by Eqs. (A.28) and (A.29) }\end{array}$ & Greek letters \\
\hline $\begin{array}{l}\text { coefficient in Eq. ( } 27 \mathrm{c}) \text { for the internal flow } \\
\text { field, given by Eqs. (A.32) }\end{array}$ & $a / k^{1 / 2}$ \\
\hline $\begin{aligned} C_{3}, D_{3} & \text { coefficients in Eq. (12c) for the internal flow } \\
& \text { field, given by Eqs. (A.19) and (A.20) }\end{aligned}$ & $\begin{array}{l}b / k^{1 / 2} \\
c / k^{1 / 2}\end{array}$ \\
\hline $\begin{array}{l}C^{\prime}, D^{\prime} \text { coefficients in Eq. (27b) for the flow field } \\
\text { inside the porous shell, given by Eqs. (A.30) } \\
\text { and (A.31) }\end{array}$ & $\begin{array}{ll}\zeta & b / c \\
\eta & \text { viscosity of the fluid, } \mathrm{kg} \mathrm{m}^{-1} \mathrm{~s}^{-1} \\
\theta, \phi & \text { angular spherical coordinates }\end{array}$ \\
\hline $\begin{array}{l}E, F, G, H \text { coefficients in Eq. (12b) for the flow field } \\
\text { inside the porous shell, given by Eqs. } \\
\text { (A.15)-(A.18) }\end{array}$ & $\begin{array}{l}\xi \quad r / k^{1 / 2} \\
\tau_{r r}, \tau_{r \theta}, \tau_{r \phi} \text { fluid stresses relevant to the surfaces of the } \\
\text { porous shell, } \mathrm{N} \mathrm{m}^{-2}\end{array}$ \\
\hline $\begin{array}{l}\text { drag force acting on the porous shell, } \mathrm{N} \\
\text { drag force acting on the porous shell in the }\end{array}$ & $\begin{array}{l}\text { Stokes stream function of the fluid flow, } \\
\mathrm{m}^{3} \mathrm{~s}^{-1}\end{array}$ \\
\hline $\begin{array}{l}\text { absence of the cavity, } \mathrm{N} \\
\text { permeability in the porous shell, } \mathrm{m}^{2}\end{array}$ & angular velocity of the porous shell, $\mathrm{s}^{-1}$ \\
\hline $\begin{array}{l}\text { normalized translational mobility of the } \\
\text { porous shell }\end{array}$ & Subscripts \\
\hline $\begin{array}{l}\text { normalized rotational mobility of the por- } \\
\text { ous shell }\end{array}$ & $\begin{array}{l}\text { fluid external to the porous shell } \\
\text { fluid inside the porous shell }\end{array}$ \\
\hline dynamic pressure distribution, $\mathrm{N} \mathrm{m}^{-2}$ & fluid internal to the porous shell \\
\hline radial spherical coordinate, $\mathrm{m}$ & \\
\hline
\end{tabular}

treated extensively in the past. Summaries for the useful knowledge in this area and some informative references can be found in Happel and Brenner (1983) and Kim and Karrila (1991).

The motion of porous particles relative to a fluid has been studied for a long time, since they can be good models for polymer coils in a solvent and for flocs of fine particles in a colloidal suspension. An approach which includes a secondorder viscous term to Darcy's equation for the flow in porous media was developed by Brinkman (1947) and Debye and Bueche (1948) independently. Sutherland and Tan (1970) used Darcy's law for the inside flow field and the Stokes equations for the outside field of a sedimenting porous sphere and concluded that it is reasonable for an isolated sphere on the assumption of immobilized fluid within the porous structure. Their result was proven incorrect by Ooms et al. (1970) and Neale et al. (1973), who used the Brinkman equation for the flow within the porous sphere and more general boundary conditions at the surface of the particle. Experimental studies on the sedimentation of porous particles have been reported by Matsumoto and Suganuma (1977) and Masliyah and Polikar (1980), whose results are in good agreement with the theoretical prediction of Neale et al. (1973).

The creeping flow of a fluid past a composite sphere having a central solid core and an outer porous shell was solved by Masliyah et al. (1987) using the Brinkman equation for the flow field inside the fluid-permeable surface layer. An analytical formula for the drag force experienced by the particle was derived as a function of the radius of the solid core, the thickness of the porous shell, and the permeability of the shell. They also measured the settling velocity of a solid sphere with attached threads and found that theoretical predictions for the composite sphere are in excellent agreement with the experimental results. Later, the boundary effect on the motion of a composite sphere perpendicular to one or two plane walls was later examined by Chen and Ye (2000). A boundary collocation method was used to study the general case where the porous shell thickness and separation distance between the particle and the wall can be arbitrary, 
and a lubrication theory was used to analyze the special case of a particle coated with a thin permeable layer in near contact with a single plane. Recently, the translational and rotational motions of a composite sphere in a concentric spherical cavity have been analyzed by Keh and Chou (2004).

On the other hand, Jones (1973) analyzed the creeping motion of a spherically symmetric porous shell using Darcy's law and an empirical boundary condition. Later on, the same problem was investigated by Qin and Kaloni (1993) and Bhatt and Sacheti (1994) using the Brinkman equation and well-defined velocity and stress boundaries. There were several features in their analytical solutions which cannot be seen from Darcy's solution.

The object of this work is to obtain insights into the boundary effects on the translational and rotational motions of an arbitrary porous spherical shell within a small pore. This type of problem is difficult to solve, due to the structural difference for hydrodynamics inside and outside the porous shell and the complexity of the actual system geometry. In order to avoid the mathematical difficulties encountered in the problem of a sphere in a cylinder (which is a widely used model for particles in pores), we choose to examine the motions of a porous spherical shell situated at the center of a spherical cavity. Although the spherical cavity geometry is an idealized abstraction of any real system, the results obtained in this geometry have been shown to be in good agreement with available solutions for the boundary effects on the partition coefficient (Giddings et al., 1968; Glandt, 1981), settling velocity (Bungay and Brenner, 1973; Happel and Brenner, 1983), and electrophoretic mobility (Zydney, 1995; Keh and Chiou, 1996) of a nonporous particle in a cylindrical pore. The spherical symmetry in this model system allows exact analytical solutions to be obtained, and the results show that the boundary effects on the motions of a porous shell can be significant in general situations.

\section{Translation of a porous spherical shell in a spherical cavity}

In this section we consider the quasisteady translational motion of a porous spherical shell of external radius $b$ and internal radius $a$, in a concentric spherical cavity of radius $c$, filled with an incompressible Newtonian fluid of viscosity $\eta$, as illustrated in Fig. 1. The porous shell is assumed to be nondeformable, and its velocity equals $U$ in the positive $z$ (axial) direction. The spherical coordinate system $(r, \theta, \phi)$ is established with its origin at the center of the porous shell or cavity. The Reynolds number is assumed to be sufficiently small so that the inertial terms in the fluid momentum equation can be neglected, in comparison with the viscous terms. Our purpose here is to determine the hydrodynamic drag force exerted on the porous shell in the presence of the cavity.

The external region $(b \leq r \leq c)$, the porous region $(a \leq r \leq b)$, and the internal region $(r \leq a)$ are denoted as regions I, II, and III, respectively. Then, the fluid flow in regions I and III is governed by the Stokes equations,

$$
\begin{aligned}
& \eta \nabla^{2} \mathbf{v}_{i}-\nabla p_{i}=\mathbf{0}, \\
& \nabla \cdot \mathbf{v}_{i}=0
\end{aligned}
$$

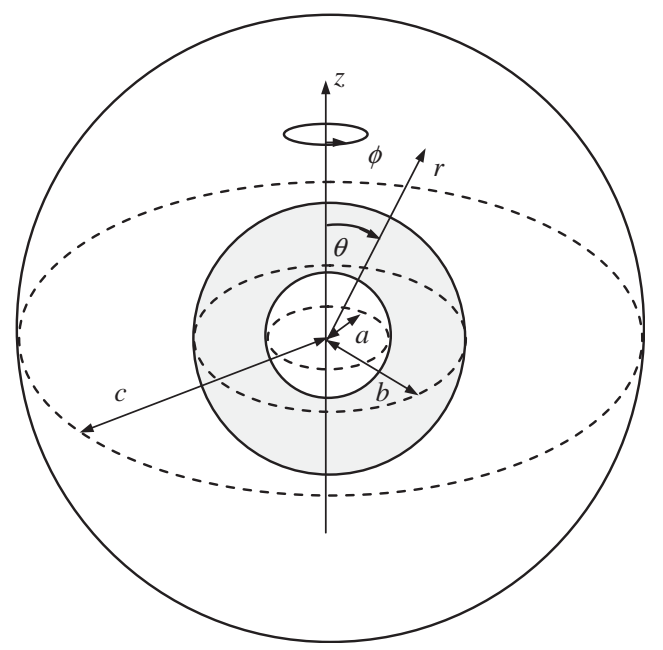

Fig. 1. Geometric sketch for the motion of a porous spherical shell in a concentric spherical cavity. 
Here, $\mathbf{v}$ is the fluid velocity field for the flow relative to the porous shell, $p$ is the corresponding dynamic pressure distribution, $i=1$ or 3 , and the subscripts 1 and 3 represent the regions I and III, respectively.

For the fluid flow within the porous shell, the relative velocity $\mathbf{v}_{2}$ and dynamic pressure $p_{2}$ are governed by the Brinkman equation, which is preferred to the Darcy equation to accommodate the boundary conditions at the particle surfaces $(r=a$ and $b)$,

$$
\eta \nabla^{2} \mathbf{v}_{2}-\frac{\eta}{k} \mathbf{v}_{2}-\nabla p_{2}=\mathbf{0}
$$

and

$$
\nabla \cdot \boldsymbol{v}_{2}=0
$$

where $k$ is the permeability of the porous shell, and the quantities with subscript 2 represent macroscopically averaged quantities pertaining to the porous medium. Here, we have assumed that the fluid has the same viscosity inside and outside the permeable shell which is reasonable according to available evidence (Koplik et al., 1983). For some model porous particles made of steel wool (in glycerin-water solution) (Matsumoto and Suganuma, 1977) and plastic foam slab (in silicon oil) (Masliyah and Polikar, 1980), experimental values of $k$ can be as large as $10^{-7} \mathrm{~m}^{2}$, while in the poly( $N$-isopropylacrylamide) hydrogel layers on latex particles in electrolyte solutions, values of $k$ were found to be about $10^{-15}-10^{-18} \mathrm{~m}^{2}$ (Makino et al., 1994).

Since the flow field is axially symmetric, it is convenient to introduce the Stokes stream functions $\Psi_{i}(r, \theta)$ which satisfy Eqs. (1b) and (2b) and are related to the velocity components in the spherical coordinate system by

$$
\begin{aligned}
& v_{r i}=-\frac{1}{r^{2} \sin \theta} \frac{\partial \Psi_{i}}{\partial \theta}, \\
& v_{\theta i}=\frac{1}{r \sin \theta} \frac{\partial \Psi_{i}}{\partial r} .
\end{aligned}
$$

Taking the curl of Eq. (1a) and applying Eq. (3) gives a fourth-order linear partial differential equation for $\Psi_{i}$,

$$
E_{s}^{4} \Psi_{i}=E_{s}^{2}\left(E_{s}^{2} \Psi_{i}\right)=0
$$

where $i=1$ or 3 , and the axisymmetric Stokes operator $E_{s}^{2}$ is given by

$$
E_{s}^{2}=\frac{\partial^{2}}{\partial r^{2}}+\frac{\sin \theta}{r^{2}} \frac{\partial}{\partial \theta}\left(\frac{1}{\sin \theta} \frac{\partial}{\partial \theta}\right) .
$$

Accordingly, Eq. (2a) can be expressed in terms of the stream function $\Psi_{2}(r, \theta)$ as

$$
E_{s}^{4} \Psi_{2}-\frac{1}{k} E_{s}^{2} \Psi_{2}=0
$$

Due to the continuity of velocity and stress components at the surfaces of the porous shell, which is physically realistic and mathematically consistent for the present problem (Neale et al., 1973; Koplik et al., 1983; Chen and Ye, 2000), the boundary conditions for the flow field are

$$
\begin{array}{lc}
r=a: & v_{r 3}=v_{r 2}, \quad v_{\theta 3}=v_{\theta 2}, \\
\tau_{r r 3}=\tau_{r r 2}, & \tau_{r \theta 3}=\tau_{r \theta_{2}}, \\
r=b: & v_{r 2}=v_{r 1}, \quad v_{\theta 2}=v_{\theta 1}, \\
\tau_{r r 2}=\tau_{r r 1}, & \tau_{r \theta 2}=\tau_{r \theta 1}, \\
r=c: & v_{r 1}=-U \cos \theta,
\end{array}
$$

Here, $\tau_{r r}$ and $\tau_{r \theta}$ are the normal and shear stresses for the fluid flow relevant to the particle surfaces. Eqs. (7)-(11) take a reference frame that the porous spherical shell is at rest and the velocity of the fluid at the cavity wall is the particle velocity in the opposite direction. Since we take the same fluid viscosity inside and outside the porous shell, use the fluid velocity continuity given by Eqs. (7) and (9), and neglect the possible osmotic effects in the porous shell, Eqs. (8a) and (10a) are equivalent to the continuity of pressure $\left(p_{3}=p_{2}\right.$ at $r=a$ and $p_{2}=p_{1}$ at $\left.r=b\right)$. 
A solution to Eqs. (4) and (6) suitable for satisfying boundary conditions on the spherical surfaces is (Masliyah et al., 1987; Keh and Chou, 2004)

$$
\begin{aligned}
& \Psi_{1}=\frac{1}{2} k U\left(A \xi^{-1}+B \xi+C_{1} \xi^{2}+D_{1} \xi^{4}\right) \sin ^{2} \theta \quad \text { if } \quad \beta \leq \xi \leq \gamma, \\
& \Psi_{2}=\frac{1}{2} k U\left[E \xi^{-1}+F \xi^{2}+G\left(\xi^{-1} \cosh \xi-\sinh \xi\right)+H\left(\xi^{-1} \sinh \xi-\cosh \xi\right)\right] \sin ^{2} \theta \quad \text { if } \quad \alpha \leq \xi \leq \beta, \\
& \Psi_{3}=\frac{1}{2} k U\left(C_{3} \xi^{2}+D_{3} \xi^{4}\right) \sin ^{2} \theta \quad \text { if } \quad \xi \leq \alpha,
\end{aligned}
$$

where the dimensionless variables $\xi=r / k^{1 / 2}, \alpha=a / k^{1 / 2}, \beta=b / k^{1 / 2}$, and $\gamma=c / k^{1 / 2}$. The dimensionless constants $A, B$, $C_{1}, D_{1}, C_{3}, D_{3}, E, F, G$ and $H$ are found from Eqs. (7)-(11) using Eq. (3). The procedure is straightforward but tedious, and the result is given in the appendix.

The drag force (in the $z$ direction) exerted by the external fluid on the porous shell with the spherical boundary $r=b$ can be determined from (Happel and Brenner, 1983)

$$
F_{d}=\pi \eta \int_{0}^{\pi} r^{3} \sin ^{3} \theta \frac{\partial}{\partial r}\left(\frac{E_{s}^{2} \Psi_{1}}{r^{2} \sin ^{2} \theta}\right) r \mathrm{~d} \theta
$$

Substitution of Eq. (12a) into the above integral results in the simple relation

$$
F_{d}=4 \pi \eta U B k^{1 / 2}
$$

where $B$ is given by Eq. (A.12) in the appendix.

In the limiting case of $\beta / \gamma=b / c=0$, the above equation becomes

$$
F_{d}^{(0)}=-6 \pi \eta b U \frac{R}{\beta S}
$$

where

$$
\begin{aligned}
R= & 2\left(3 \beta w_{2}-\alpha w_{1}\right) \cosh (\beta-\alpha)+2\left(3 w_{2}-\alpha \beta w_{1}\right) \sinh (\beta-\alpha) \\
S= & 60 \alpha^{3}-3\left[\left(\alpha^{2}+15\right)\left(4 \alpha^{3}+\alpha-w_{3}\right)-\alpha^{2} w_{3}-10 \alpha\left(5 \alpha^{2}-3\right)\right] \cosh (\beta-\alpha)-\left[\left(\alpha^{2}+15\right)\left(2 \alpha^{4}+9-\alpha w_{3}\right)-30 \alpha w_{3}+9 \alpha^{2}\right] \\
& \times \sinh (\beta-\alpha)
\end{aligned}
$$

with

$$
\begin{aligned}
& w_{1}=\beta^{3}\left(\alpha^{2}+45\right)-\alpha^{3}\left(\alpha^{2}+15\right), \\
& w_{2}=\beta^{3}\left(2 \alpha^{2}+15\right)-\alpha^{3}\left(2 \alpha^{2}+5\right), \\
& w_{3}=\beta\left(2 \beta^{2}+3\right) .
\end{aligned}
$$

The formula given by Eq. (15) is the reduced result for the translation of an isolated porous spherical shell in an unbounded fluid obtained by Bhatt and Sacheti (1994).

Through the use of Eqs. (14) and (15), the normalized translational mobility of a porous spherical shell in a concentric spherical cavity can be expressed as

$$
M=\frac{F_{d}^{(0)}}{F_{d}}=-\frac{3 R}{2 B S} .
$$

Note that $M=1$ as $\beta / \gamma=0$ and $0 \leq M<1$ as $0<\beta / \gamma \leq 1$. The presence of the cavity wall always enhances the hydrodynamic drag on the porous shell since the fluid flow vanishes at the wall as required by Eq. (11).

When $k=0$, we have $F_{d}^{(0)}=-6 \pi \eta b U$ (Stokes' law), and Eq. (18) reduces to

$$
M=\left(1-\frac{9}{4} \zeta+\frac{5}{2} \zeta^{3}-\frac{9}{4} \zeta^{5}+\zeta^{6}\right)\left(1-\zeta^{5}\right)^{-1},
$$

where $\zeta=b / c$. This is the result for the translation of a solid sphere of radius $b$ in a cavity of radius $c$ (Happel and Brenner, 1983). 
When $a=0$, Eqs. (15) and (18) become

$$
\begin{aligned}
& F_{d}^{(0)}=-6 \pi \eta b U \frac{2 \beta^{2}(\beta-\tanh \beta)}{2 \beta^{3}+3(\beta-\tanh \beta)}, \\
& M=\frac{(\beta \cosh \beta-\sinh \beta)\left(s_{21} \beta \cosh \beta-3 s_{23} \sinh \beta\right)}{2 \gamma\left[\beta\left(2 \beta^{2}+3\right) \cosh \beta-3 \sinh \beta\right]\left(s_{8} \sinh \beta-s_{5} \beta \cosh \beta\right)},
\end{aligned}
$$

where the dimensionless parameters $s_{5}, s_{8}, s_{21}$, and $s_{23}$ are defined by Eq. (A.22) in the appendix. The hydrodynamic drag and normalized mobility given by Eqs. (20) and (21) describe the translation of a porous (permeable) sphere of radius $b$ in an unbounded fluid (Neale et al., 1973) and in a cavity of radius $c$, respectively. In the limiting case of $\beta \rightarrow \infty$ (or $k=0$ ), Eq. (20) reduces to the Stokes law $F_{d}^{(0)}=-6 \pi \eta b U$, while in the limit of the singular case $\beta=0$ (or $k \rightarrow \infty)$, it becomes $F_{d}^{(0)}=0$.

The variation of the normalized mobility $M$ given by Eq. (21) for the translation of a porous sphere (with $a=0$ or $\alpha / \beta=0)$ at the center of a spherical cavity with the separation parameter $\beta / \gamma$ for various values of $\beta$ from zero to infinity is presented in Fig. 2. The separation parameter $\beta / \gamma(=b / c)$, reflecting the extent of closeness between the particle and the cavity wall, ranges from 0 (far apart) to 1 (in contact). The curve with $\beta=0$ (or $k \rightarrow \infty$ ) represents the result for the singular case of a porous sphere with no resistance to the fluid flow, while the curve with $\beta \rightarrow \infty$ (or $k=0$ ) denotes the result for a solid particle. As expected, the normalized mobility $M$ equals unity as $\beta=0$ for any value of $\beta / \gamma$ (with $F_{d}=F_{d}^{(0)}=0$ for a given particle velocity $U$ ) and is a monotonic decreasing function of $\beta / \gamma$ for any given value of $\beta>0$. Obviously, the boundary effect on the particle mobility (or drag force) is stronger when the permeability $k$ of the particle is smaller (or $\beta$ is greater). For $\beta<1$, the particle mobility varies slowly with the separation parameter $\beta / \gamma$, compared with the case of lower permeability (or greater $\beta$ ). This weak interaction can be explained by the fact that, instead of bypassing, the fluid can easily flow through a porous particle with a high permeability, leading to a great reduction in the resistant force. For $\beta>10$, the value of the particle mobility is quite close to that of a solid particle [with $\beta \rightarrow \infty$ and $M$ given by Eq. (19)] when $\beta / \gamma$ is small, but the difference becomes more significant as the particle gets closer to the wall. This implies that, far from the wall, a porous particle with a low permeability behaves like a solid one with most fluid flowing over it. When the porous particle and cavity wall become sufficiently close together, a large pressure gradient is developed in between to drive more fluid to permeate through the porous medium (Chen and Ye, 2000). Interestingly, for situations with a finite value of $\beta$, the particle mobility does not vanish even for the singular case as the particle touches the cavity wall (i.e. as $\beta / \gamma=1$ ).

After understanding the boundary effect on the translation of a porous sphere, we now examine the general case of a translating porous spherical shell in a concentric spherical cavity. Figs. 3(a) and (b) show the normalized mobility $M$ as a function of $\beta / \gamma$ over the entire ranges of the separation and the parameter $\alpha / \beta$ for the cases of $\beta=5$ and 1 , respectively. Again, $M$ decreases monotonically with an increase in $\beta / \gamma$ for fixed values of $\alpha / \beta$ and $\beta$ and with an

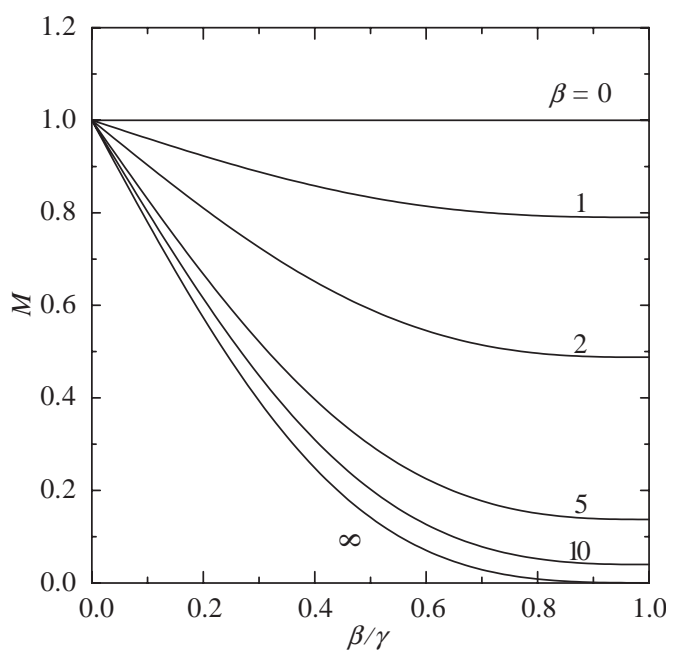

Fig. 2. Plots of the normalized translational mobility $M$ for a porous sphere $(a=0)$ in a concentric spherical cavity versus the separation parameter $\beta / \gamma$ for various values of $\beta$. 

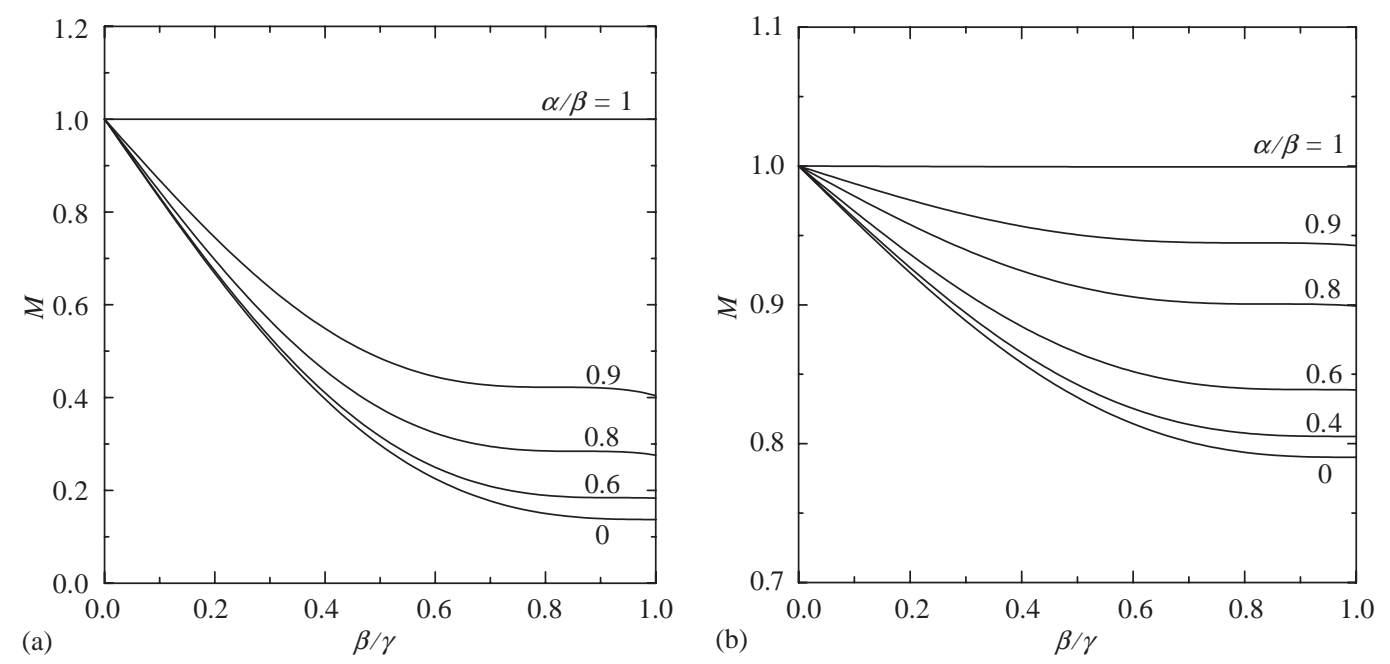

Fig. 3. Plots of the normalized translational mobility $M$ for a porous spherical shell in a concentric spherical cavity versus the separation parameter $\beta / \gamma$ for various values of $\alpha / \beta$ : (a) $\beta=5$; (b) $\beta=1$.

increase in $\beta$ for constant values of $\alpha / \beta$ and $\beta / \gamma$. As expected, the curves for the singular case with $\alpha / \beta=1$ lead to $M=1$ (with $F_{d}=F_{d}^{(0)}=0$ for a given particle velocity $U$ ) and the curves with $\alpha / \beta=0$ represent the result for a porous sphere [given by Eq. (21)]. All the other curves for a porous spherical shell lie between these upper and lower bounds and $M$ is a monotonic increasing function of $\alpha / \beta$ for given values of $\beta$ and $\beta / \gamma$. Namely, the hydrodynamic drag acting on the porous shell is reduced as its thickness becomes smaller for a given shell size, permeability, and separation distance. It can be seen that, for the case of $\beta=5$, the behavior of a porous spherical shell with $\alpha / \beta=0.6$ can be roughly approximated by that of a porous sphere of equal size (or $\beta / \gamma$ ) and permeability when $\beta / \gamma<0.5$. This is because, when a porous shell has a low to moderate permeability, it is difficult for the fluid to penetrate deep to reach its inner surface as long as the shell is sufficiently thick and the cavity wall is not too close. Thus, the internal fluid (in region III) remains oblivious of the external motion. However, this approximation is no longer valid for a porous shell with a large permeability, as for the case of $\beta=1$ shown in Fig. 3(b). Again, for situations with a finite value of $\beta$, the particle mobility is not necessarily equal to zero for the singular case as $\beta / \gamma=1$.

\section{Rotation of a porous spherical shell in a spherical cavity}

We now consider the steady rotational motion of a porous spherical shell of external radius $b$ and internal radius $a$ located at the center of a spherical cavity of radius $c$. The angular velocity of the porous shell is $\Omega$ in the positive $z$ (axial) direction and the Reynolds number is vanishingly small. The objective in this section is to obtain the hydrodynamic torque acting on the porous shell in the presence of the cavity.

The fluid flow fields outside and inside the porous shell are still governed by Eqs. (1) and (2) respectively, and they must be solved subject to the following boundary conditions resulting from the continuity of velocity and stress components:

$$
\begin{aligned}
& r=a: \quad v_{\phi 3}=v_{\phi 2}, \quad \tau_{r \phi 3}=\tau_{r \phi 2}, \\
& r=b: \quad v_{\phi 2}=v_{\phi 1}, \quad \tau_{r \phi 2}=\tau_{r \phi 1}, \\
& r=c: \quad v_{\phi 1}=-\Omega c \sin \theta,
\end{aligned}
$$

where $v_{\phi}$ is the $\phi$-component of the fluid velocity field and $\tau_{r \phi}$ is the shear stress for the rotational fluid flow relevant to the surfaces of the porous shell. Obviously, the $r$ and $\theta$ components of the fluid velocity disappear and the fluid dynamic 
pressure is constant everywhere. Eqs. (22)-(26) take a reference frame that the porous shell is at rest and the angular velocity of the cavity wall is that of the shell in the opposite direction.

A solution to Eqs. (1) and (2) suitable for satisfying the boundary conditions (22)-(26) is (Keh and Chou, 2004)

$$
\begin{aligned}
& v_{\phi 1}=k^{1 / 2} \Omega\left(A^{\prime} \xi^{-2}+B_{1}^{\prime} \xi\right) \sin \theta \quad \text { if } \beta \leq \xi \leq \gamma, \\
& v_{\phi 2}=k^{1 / 2} \Omega\left[C^{\prime}\left(\xi^{-2} \cosh \xi-\xi^{-1} \sinh \xi\right)+D^{\prime}\left(\xi^{-2} \sinh \xi-\xi^{-1} \cosh \xi\right)\right] \sin \theta \quad \text { if } \alpha \leq \xi \leq \beta, \\
& v_{\phi 3}=k^{1 / 2} \Omega B_{3}^{\prime} \xi \sin \theta \quad \text { if } \xi \leq \alpha,
\end{aligned}
$$

where the dimensionless variables $\xi, \alpha, \beta$, and $\gamma$ were defined right after Eq. (12). The constants $A^{\prime}, B_{1}^{\prime}, B_{3}^{\prime}, C^{\prime}$, and $D^{\prime}$ can be determined from Eqs. (22)-(26), and the result is given in the appendix.

After the fluid velocity field is solved, the torque (in the $z$ direction) exerted on the rotating porous spherical shell about its center by the external fluid can be obtained as

$$
T_{d}=-8 \pi \eta \Omega A_{1}^{\prime} k^{3 / 2},
$$

where $A_{1}^{\prime}$ is given by Eq. (A.28). In the limit of $\beta / \gamma=b / c=0$, Eq. (28) becomes

$$
T_{d}^{(0)}=-8 \pi \eta b^{3} \Omega \frac{R^{\prime}}{\beta^{2} S^{\prime}},
$$

the reduced result for the rotation of a porous spherical shell in an unbounded fluid, where

$$
\begin{aligned}
& R^{\prime}=3\left[\alpha\left(\beta^{2}+3\right)-\beta\left(\alpha^{2}+3\right)\right] \cosh (\beta-\alpha)+\left[\left(\alpha^{2}+3\right)\left(\beta^{2}+3\right)-9 \alpha \beta\right] \sinh (\beta-\alpha), \\
& S^{\prime}=3 \alpha \cosh (\beta-\alpha)+\left(\alpha^{2}+3\right) \sinh (\beta-\alpha) .
\end{aligned}
$$

The normalized rotational mobility of a porous spherical shell in a concentric spherical cavity can be expressed as

$$
N=\frac{T_{d}^{(0)}}{T_{d}}=\frac{\beta R^{\prime}}{A^{\prime} S^{\prime}}
$$

The presence of the no-slip cavity wall always enhances the hydrodynamic torque on the rotating porous shell. Thus, $0 \leq N \leq 1$ for the entire range of $\beta / \gamma(N=1$ as $\beta / \gamma=0)$.

When $k=0, T_{d}^{(0)}=-8 \pi \eta b^{3} \Omega$, and Eq. (31) reduces to

$$
N=1-\zeta^{3},
$$

where $\zeta=b / c$. This is the result for the rotation of a solid sphere of radius $b$ in a cavity of radius $c$.

When $a=0$, Eqs. (29) and (31) become

$$
\begin{aligned}
& T_{d}^{(0)}=-8 \pi \eta b^{3} \Omega\left(1+3 \beta^{-2}-3 \beta^{-1} \operatorname{coth} \beta\right), \\
& N=1-\frac{\beta^{3}}{\gamma^{3}}\left(1+3 \beta^{-2}-3 \beta^{-1} \operatorname{coth} \beta\right) .
\end{aligned}
$$

The hydrodynamic torque and normalized mobility predicted by Eqs. (33) and (34) describe the rotation of a porous sphere of radius $b$ in an unbounded fluid and in a cavity of radius $c$, respectively. In the limiting case of $\beta \rightarrow \infty$ (or $k=0$ ), Eq. (33) reduces to $T_{d}^{(0)}=-8 \pi \eta b^{3} \Omega$, while in the limit of the singular case $\beta=0$ (or $k \rightarrow \infty$ ), it results in $T_{d}^{(0)}=0$.

Fig. 4 shows the plot of the normalized rotational mobility $N$ for a porous sphere at the center of a spherical cavity versus the separation parameter $\beta / \gamma$ for various values of $\beta$ over the entire ranges. Analogous to the result of the translational mobility $M$ of the particle, the rotational mobility $N$ equals unity as $\beta=0$ for all values of $\beta / \gamma$ (with $T_{d}=T_{d}^{(0)}=0$ for a given angular velocity $\Omega$ of the particle) and decreases monotonically with an increase in the value of $\beta / \gamma$ for a specified value of $\beta>0$. The boundary effect on the rotational mobility (or hydrodynamic torque) of the permeable particle is stronger when the permeability $k$ is smaller (or $\beta$ is greater). For $\beta<1$, the rotational mobility is not a sensitive function of $\beta / \gamma$ (except as $\beta / \gamma \rightarrow 1$ ), compared with the result for a lower permeability (or greater $\beta$ ). For $\beta>10$, the value of the rotational mobility of the porous sphere is close to that of a solid particle [with $k=0$ or $\beta \rightarrow \infty$ and $N$ given by Eq. (32)] when $\beta / \gamma$ is small, while the difference is more significant as $\beta / \gamma \rightarrow 1$. When a particle with a finite value of $\beta$ is in contact with the cavity wall $(\beta / \gamma=1)$, its rotational mobility does not vanish for this singular case. 


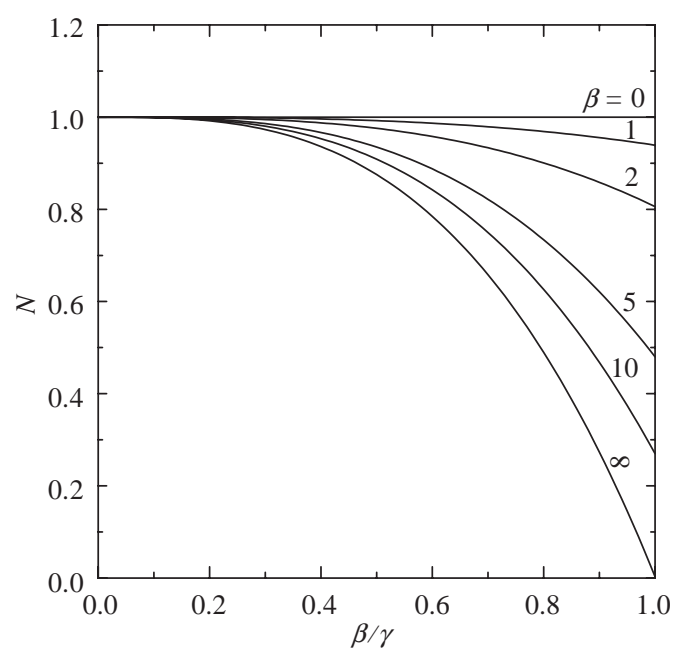

Fig. 4. Plots of the normalized rotational mobility $N$ for a porous sphere $(a=0)$ in a concentric spherical cavity versus the separation parameter $\beta / \gamma$ for various values of $\beta$.
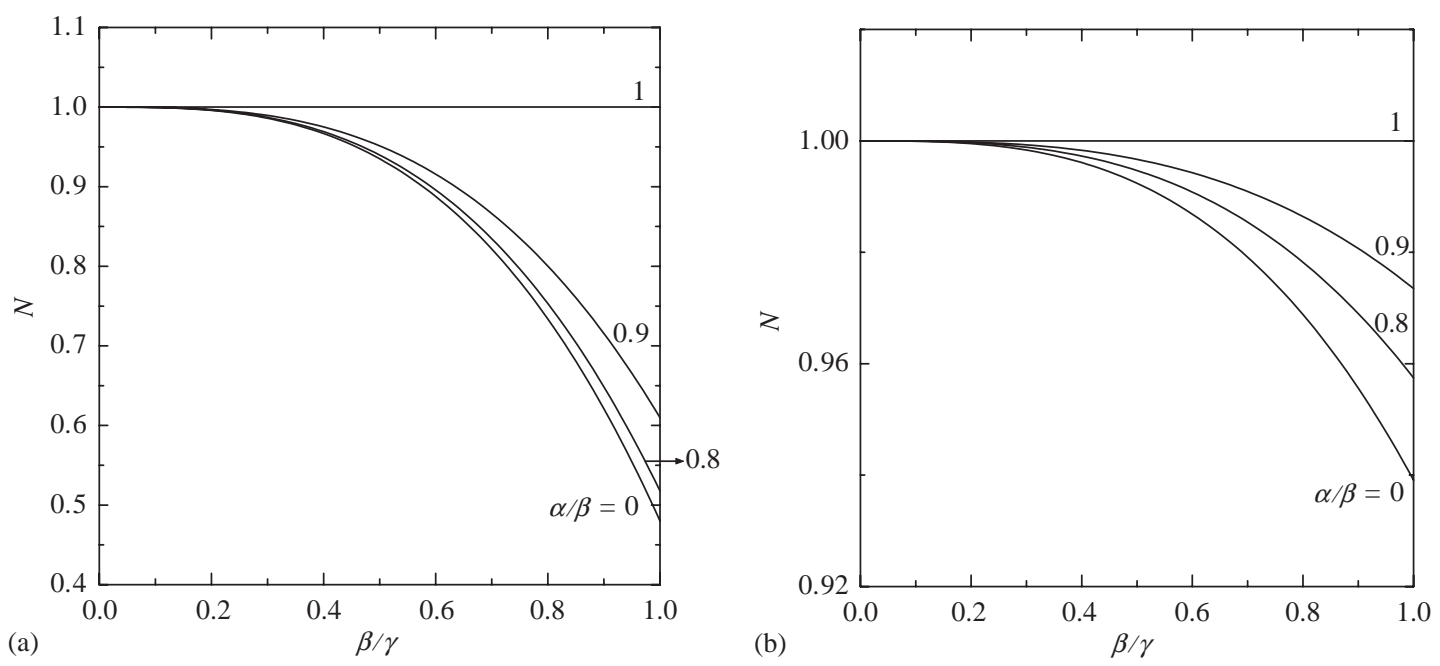

Fig. 5. Plots of the normalized rotational mobility $N$ for a porous spherical shell in a concentric spherical cavity versus the separation parameter $\beta / \gamma$ for various values of $\alpha / \beta$ : (a) $\beta=5$ and (b) $\beta=1$.

In Figs. 5(a) and (b), we present the results of the normalized rotational mobility $N$ for a porous spherical shell in a concentric spherical cavity as a function of the parameters $\beta / \gamma$ and $\alpha / \beta$ over the entire ranges for the cases of $\beta=5$ and 1 , respectively. Again, $N$ decreases monotonically with an increase in $\beta / \gamma$ for given values of $\beta$ and $\alpha / \beta$ and with an increase in $\beta$ for fixed values of $\alpha / \beta$ and $\beta / \gamma$. As expected, the curves for the singular case with $\alpha / \beta=1$ give $N=1$ (with $T_{d}=T_{d}^{(0)}=0$ for a given angular velocity $\Omega$ of the particle), and the curves with $\alpha / \beta=0$ represent the result for the rotation of a porous sphere. All the other curves for a porous spherical shell locate between these upper and lower bounds and $N$ increases monotonically with an increase in $\alpha / \beta$ for specified values of $\beta$ and $\beta / \gamma$. For a porous shell with a finite value of $\beta$, its rotational mobility does not vanish for the singular case as $\beta / \gamma=1$. For the case of $\beta=5$, the normalized rotational mobility of a porous spherical shell with $\alpha / \beta=0.8$ can be well approximated by that of a porous sphere of equal size, permeability, and separation distance from the cavity wall. As illustrated in Fig. 5(b), however, this approximation is no longer valid for a porous shell with a large permeability. 


\section{Concluding remarks}

In this work, the quasisteady translation and steady rotation of a porous spherical shell (which can reduce to a solid sphere and a porous sphere in the limiting cases) in a concentric spherical cavity filled with an incompressible Newtonian fluid have been investigated theoretically in the creeping flow regime. The Stokes and Brinkman equations for the fluid flow field applicable to these axisymmetric motions are analytically solved and the hydrodynamic drag force and torque exerted on the porous shell as functions of the parameters $\alpha / \beta(=a / b), \beta / \gamma(=b / c)$, and $\left(\beta=b / k^{1 / 2}\right)$ are obtained in the closed-form expressions (14) and (28). It has been found that, for a specified geometry (fixed values of $\alpha / \beta$ and $\beta / \gamma)$, the wall-corrected translational and rotational mobilities of the porous shell, normalized by their corresponding values in the absence of the cavity wall, are monotonic decreasing functions of the parameter $\beta$ (or increasing functions of the permeability $k$ ) of the porous shell. For given values of $\alpha / \beta$ and $\beta$, these normalized mobilities decrease monotonically with an increase in the separation parameter $\beta / \gamma$. The analysis assumes that the porous shell is non-deformable. The results would be very different, particularly in the case where the porous shell just fits within the cavity, if it were able to deform in response to the flow (as might be expected for a shell composed of entangled polymer).

Our results, which provide useful insights into the actual phenomena regarding the creeping motions of a porous particle in a small pore, show that the boundary effect of the cavity wall on these motions can be significant in appropriate situations. Also, these results will serve as useful limiting solutions against which the results of more sophisticated models (which account for shell deformability, for instance) may be benchmarked. More detailed analyses of the fluid flow for a porous particle in open and closed cylindrical pores will still be required to quantify the actual behavior in this system and to determine the overall applicability of the results obtained in this work for the spherical cavity to more realistic pore geometries.

\section{Acknowledgements}

Part of this research was supported by the National Science Council of the Republic of China.

\section{Appendix}

For conciseness, algebraic equations for the determination of the coefficients in Eqs. (12) and (27) as well as their solutions are presented in this appendix.

Applying the boundary conditions given by Eqs. (7)-(11) to the general solution given by Eq. (12) for the translation of a porous spherical shell in a concentric spherical cavity, one obtains

$$
\begin{aligned}
& C_{3} \alpha^{3}+D_{3} \alpha^{5}=E+F \alpha^{3}-G(\alpha \sinh \alpha-\cosh \alpha)+H(\sinh \alpha-\alpha \cosh \alpha), \\
& -2 C_{3} \alpha^{3}-4 D_{3} \alpha^{5}=E-2 F \alpha^{3}-G\left[\alpha \sinh \alpha-\left(\alpha^{2}+1\right) \cosh \alpha\right] \\
& -H\left[\alpha \cosh \alpha-\left(\alpha^{2}+1\right) \sinh \alpha\right] \text {, } \\
& 6 D_{3} \alpha^{5}=6 E+3\left(\alpha^{2}+2\right)(G \cosh \alpha+H \sinh \alpha)-\alpha\left(\alpha^{2}+6\right)(G \sinh \alpha+H \cosh \alpha), \\
& 20 D_{3} \alpha^{3}=E-2 F \alpha^{3}, \\
& A+B \beta^{2}+C_{1} \beta^{3}+D_{1} \beta^{5}=E+F \beta^{3}-G(\beta \sinh \beta-\cosh \beta) \\
& +H(\sinh \beta-\beta \cosh \beta) \text {, } \\
& A-B \beta^{2}-2 C_{1} \beta^{3}-4 D_{1} \beta^{5}=E-2 F \beta^{3}-G\left[\beta \sinh \beta-\left(\beta^{2}+1\right) \cosh \beta\right] \\
& -H\left[\beta \cosh \beta-\left(\beta^{2}+1\right) \sinh \beta\right] \text {, } \\
& 6 A+6 D_{1} \beta^{5}=6 E+3\left(\beta^{2}+2\right)(G \cosh \beta+H \sinh \beta) \\
& -\beta\left(\beta^{2}+6\right)(G \sinh \beta+H \cosh \beta) \text {, }
\end{aligned}
$$




$$
\begin{aligned}
& 2 B+20 D_{1} \beta^{3}=E-2 F \beta^{3}, \\
& A+B \gamma^{2}+C_{1} \gamma^{3}+D_{1} \gamma^{5}=\gamma^{3}, \\
& A-B \gamma^{2}-2 C_{1} \gamma^{3}-4 D_{1} \gamma^{5}=-2 \gamma^{3} .
\end{aligned}
$$

The above simultaneous algebraic equations can be solved to yield the ten unknown constants as

$$
\begin{aligned}
& A=2 \Delta \beta^{2} \gamma^{3}\left\{30 \alpha^{3} \beta\left(\gamma^{3}-10 \beta^{3}\right)\right. \\
& +3\left[\alpha^{3} \beta\left(2 \alpha^{2}+5\right) s_{1}-\alpha^{4} s_{0} s_{2}-\beta^{2}\left(2 \alpha^{2}+15\right) s_{3}+\alpha \beta\left(\alpha^{2}+45\right) s_{4}\right] \cosh (\beta-\alpha) \\
& \left.+\left[\alpha^{4} \beta s_{0} s_{1}-9 \alpha^{3}\left(2 \alpha^{2}+5\right) s_{2}-\alpha \beta^{2}\left(\alpha^{2}+45\right) s_{3}+9 \beta\left(2 \alpha^{2}+15\right) s_{4}\right] \sinh (\beta-\alpha)\right\}, \\
& B=6 \Delta \gamma\left\{300 \alpha^{3} \beta^{6}\right. \\
& +\left[3 \beta^{4}\left(2 \alpha^{2}+15\right) s_{5}+\alpha^{4} s_{0} s_{6}-3 \alpha^{3} \beta\left(2 \alpha^{2}+5\right) s_{7}-\alpha \beta^{3}\left(\alpha^{2}+45\right) s_{8}\right] \cosh (\beta-\alpha) \\
& \left.+\left[\alpha \beta^{4}\left(\alpha^{2}+45\right) s_{5}+3 \alpha^{3}\left(2 \alpha^{2}+5\right) s_{6}-\alpha^{4} \beta s_{0} s_{7}-3 \beta^{3}\left(2 \alpha^{2}+15\right) s_{8}\right] \sinh (\beta-\alpha)\right\} \text {, } \\
& C_{1}=\Delta \gamma\left\{30 \alpha^{3}\left(s_{10}-45 \beta^{5}\right)\right. \\
& +3\left\{\beta\left(2 \alpha^{2}+15\right)\left[s_{9}+4 \beta^{2} \gamma^{5}+30 \beta^{3}\left(\gamma^{2}-9\right)\right]-2 \alpha^{5} s_{10}-\alpha^{3} s_{11}-45 \alpha s_{12}\right. \\
& \left.-5 \alpha^{4} \beta^{2} s_{0} s_{14}\right\} \cosh (\beta-\alpha) \\
& +\left\{135 \beta^{7}\left(2 \alpha^{2}+15\right)+\alpha \beta\left(\alpha^{2}+45\right) s_{9}+45 \alpha^{3} \beta^{2} \gamma^{2}\left(2 \alpha^{2}+5\right)-2 \gamma^{5} s_{27}\right. \\
& +9 \beta^{5} s_{28}-15 \alpha \beta^{4} s_{29} \\
& \left.\left.+\beta^{3}\left[4 \alpha \gamma^{5}\left(\alpha^{2}+45\right)-5 \alpha^{4} s_{0}\left(\gamma^{2}-36\right)-90\left(2 \alpha^{2}+15\right)\left(\gamma^{2}-9\right)\right]\right\} \sinh (\beta-\alpha)\right\}, \\
& D_{1}=3 \Delta \gamma\left\{30 \alpha^{3} \beta^{3}\right. \\
& +\left[3 \beta^{4}\left(2 \alpha^{2}+15\right)\left(6-s_{13}\right)+\alpha^{4} s_{0} s_{14}-45 \alpha \beta^{3}\left(s_{14}+6\right)+6 \alpha^{5} \beta s_{13}\right. \\
& \left.-\alpha^{3} \beta s_{25}\right] \cosh (\beta-\alpha) \\
& +\left[45 \alpha \beta^{4}\left(6-s_{13}\right)-3 \beta^{3}\left(2 \alpha^{2}+15\right)\left(s_{14}+6\right)+6 \alpha^{5} s_{14}+\alpha^{4} \beta s_{0} s_{13}\right. \\
& \left.\left.+\alpha^{3} s_{26}\right] \sinh (\beta-\alpha)\right\} \\
& E=12 \Delta \alpha^{3} \gamma\left\{5 \beta^{3} s_{15}-\left[3 \beta\left(2 \alpha^{2}+5\right) s_{16}-\alpha s_{0} s_{17}\right] \cosh (\beta-\alpha)\right. \\
& \left.+\left[3\left(2 \alpha^{2}+5\right) s_{17}-\alpha \beta s_{0} s_{16}\right] \sinh (\beta-\alpha)\right\}, \\
& F=6 \Delta \gamma\left\{5 \alpha^{3} s_{18}-\left[3 \beta\left(2 \alpha^{2}+15\right) s_{16}-\alpha\left(\alpha^{2}+45\right) s_{17}\right] \cosh (\beta-\alpha)\right. \\
& \left.+\left[3\left(2 \alpha^{2}+15\right) s_{17}-\alpha \beta\left(\alpha^{2}+45\right) s_{16}\right] \sinh (\beta-\alpha)\right\}, \\
& G=6 \Delta \gamma\left\{\left[\alpha^{4} s_{0} s_{18}-\alpha \beta^{3}\left(\alpha^{2}+45\right) s_{15}\right] \cosh \alpha+30 \alpha^{3} \beta s_{16} \cosh \beta\right. \\
& \left.+3\left[\beta^{3}\left(2 \alpha^{2}+15\right) s_{15}-\alpha^{3}\left(2 \alpha^{2}+5\right) s_{18}\right] \sinh \alpha-30 \alpha^{3} s_{17} \sinh \beta\right\}, \\
& H=6 \Delta \gamma\left\{3\left[\alpha^{3}\left(2 \alpha^{2}+5\right) s_{18}-\beta^{3}\left(2 \alpha^{2}+15\right) s_{15}\right] \cosh \alpha+30 \alpha^{3} s_{17} \cosh \beta\right. \\
& \left.+\left[\beta^{3}\left(\alpha^{2}+45\right) s_{15}-\alpha^{3} s_{0} s_{18}\right] \sinh \alpha-30 \alpha^{2} \beta s_{16} \sinh \beta\right\}, \\
& C_{3}=3 \Delta \gamma\left\{\alpha^{5} s_{18}-\beta^{3}\left(\alpha^{2}-30\right) s_{15}\right. \\
& +6\left[-\beta\left(7 \alpha^{2}+15\right) s_{16}+\alpha\left(2 \alpha^{2}+15\right) s_{17}\right] \cosh (\beta-\alpha) \\
& \left.-6\left[\alpha \beta\left(2 \alpha^{2}+15\right) s_{16}-\left(7 \alpha^{2}+15\right) s_{17}\right] \sinh (\beta-\alpha)\right\},
\end{aligned}
$$




$$
\begin{aligned}
D_{3}= & 3 \Delta \gamma\left\{\beta^{3} s_{15}-\alpha^{3} s_{18}+6\left[\beta s_{16}-\alpha s_{17}\right] \cosh (\beta-\alpha)\right. \\
& \left.+6\left[\alpha \beta s_{16}-s_{17}\right] \sinh (\beta-\alpha)\right\},
\end{aligned}
$$

where

$$
\begin{aligned}
& \Delta=\left\{60 \alpha^{3} s_{22}+3\left[\alpha^{4} s_{0} s_{19}-2 \alpha^{5} s_{20}+\beta\left(2 \alpha^{2}+15\right) s_{21}-45 \alpha s_{23}-\alpha^{3} s_{24}\right] \cosh (\beta-\alpha)\right. \\
& \left.+\left[18 \alpha^{5} s_{19}-\alpha^{4} s_{0} s_{20}+45 \alpha \beta s_{21}-9\left(2 \alpha^{2}+15\right) s_{23}+\alpha^{3}\left(45 s_{22}-\beta s_{24}\right)\right] \sinh (\beta-\alpha)\right\}^{-1}, \\
& s_{0}=\alpha^{2}+15, \quad s_{1}=\beta^{3}+45 \beta-\gamma^{3}, \quad s_{2}=2 \beta^{3}+15 \beta-\gamma^{3}, \\
& s_{3}=\beta^{5}+15 \beta^{3}-\beta^{2} \gamma^{3}-6 \gamma^{3}, \quad s_{4}=2 \beta^{5}+5 \beta^{3}-\beta^{2} \gamma^{3}-2 \gamma^{3}, \\
& s_{5}=\beta^{5}+15 \beta^{3}-\gamma^{5}, \quad s_{6}=6 \beta^{5}+45 \beta^{3}-\gamma^{5}, \\
& s_{7}=\beta^{5}+45 \beta^{3}-\gamma^{5}, \quad s_{8}=6 \beta^{5}+15 \beta^{3}-\gamma^{5}, \\
& s_{9}=6 \gamma^{5}-9 \beta^{7}+\beta^{5}\left(5 \gamma^{2}-126\right), \quad s_{10}=4 \gamma^{5}-9 \beta^{5}+5 \beta^{3}\left(\gamma^{2}-36\right) \text {, } \\
& s_{11}=22 \gamma^{5}-15 \beta^{7}+\beta^{5}\left(5 \gamma^{2}-117\right)+5 \beta^{3}\left(7 \gamma^{2}-198\right) \text {, } \\
& s_{12}=2 \gamma^{5}-15 \beta^{7}+\beta^{5}\left(5 \gamma^{2}-72\right)+10 \beta^{3}\left(\gamma^{2}-9\right) \text {, } \\
& s_{13}=\gamma^{2}-\beta^{2} \text {, } \\
& s_{14}=3 \beta^{2}-\gamma^{2}, \\
& s_{15}=3 \beta^{5}-5 \beta^{3} \gamma^{2}+2 \gamma^{5} \text {, } \\
& s_{17}=21 \beta^{5}-5 \beta^{3}\left(\gamma^{2}-9\right)-\gamma^{5} \text {, } \\
& s_{19}=8 \beta^{5}-15 \beta^{4} \gamma+60 \beta^{3}+10 \beta^{2} \gamma^{3}-3 \gamma^{5} \text {, } \\
& s_{16}=6 \beta^{5}-5 \beta^{3}\left(\gamma^{2}-9\right)-\gamma^{5} \text {, } \\
& s_{18}=3 \beta^{5}-5 \beta^{3}\left(\gamma^{2}+18\right)+2 \gamma^{5} \text {, } \\
& s_{20}=4 \beta^{6}-9 \beta^{5} \gamma+180 \beta^{4}+10 \beta^{3} \gamma\left(\gamma^{2}-18\right)-9 \beta \gamma^{5}+4 \gamma^{6} \text {, } \\
& s_{21}=4 \beta^{8}-9 \beta^{7} \gamma+60 \beta^{6}+2 \beta^{5} \gamma\left(5 \gamma^{2}-63\right)-3 \beta^{3} \gamma\left(3 \gamma^{4}-20 \gamma^{2}+90\right)+4 \beta^{2} \gamma^{6}+6 \gamma^{6} \text {, } \\
& s_{22}=20 \beta^{6}-27 \beta^{5} \gamma+5 \beta^{3} \gamma\left(\gamma^{2}-18\right)+2 \gamma^{6} \text {, } \\
& s_{23}=8 \beta^{8}-15 \beta^{7} \gamma+20 \beta^{6}+2 \beta^{5} \gamma\left(5 \gamma^{2}-36\right)-\beta^{3} \gamma\left(3 \gamma^{4}-20 \gamma^{2}+90\right)+2 \gamma^{6} \text {, } \\
& s_{24}=8 \beta^{8}-15 \beta^{7} \gamma+40 \beta^{6}+900 \beta^{4}-45 \beta \gamma^{5}+22 \gamma^{6}+\beta^{5} \gamma\left(10 \gamma^{2}-117\right) \\
& -\beta^{3} \gamma\left(3 \gamma^{4}-70 \gamma^{2}+990\right) \\
& s_{25}=3 \beta^{4}-15 \gamma^{2}-\beta^{2}\left(\gamma^{2}-21\right) \text {, } \\
& s_{26}=\beta^{6}+45 \beta^{2}-15 \gamma^{2}-\beta^{4}\left(\gamma^{2}-6\right), \\
& s_{27}=2 \alpha^{6}+30 \alpha^{4}+18 \alpha^{2}+135 \text {, } \\
& s_{28}=\alpha^{6}+15 \alpha^{4}+1080-75 \gamma^{2}-2 \alpha^{2}\left(5 \gamma^{2}-72\right) \text {, } \\
& s_{29}=18 \alpha^{4}-\alpha^{2}\left(2 \gamma^{2}-63\right)-90\left(\gamma^{2}-9\right) \text {. }
\end{aligned}
$$

Application of the boundary conditions (22)-(26) to the general solution (27) for the rotation of a porous spherical shell in a concentric spherical cavity yields

$$
\begin{aligned}
& B_{3}^{\prime} \alpha=C^{\prime}\left(\alpha^{-2} \cosh \alpha-\alpha^{-1} \sinh \alpha\right)+D^{\prime}\left(\alpha^{-2} \sinh \alpha-\alpha^{-1} \cosh \alpha\right), \\
& 0=\left[C^{\prime}\left(\alpha^{2}+3\right)-3 D^{\prime} \alpha\right] \cosh \alpha-\left[3 C^{\prime} \alpha-D^{\prime}\left(\alpha^{2}+3\right)\right] \sinh \alpha, \\
& A^{\prime} \beta^{-2}+B_{1}^{\prime} \beta=C^{\prime}\left(\beta^{-2} \cosh \beta-\beta^{-1} \sinh \beta\right)+D^{\prime}\left(\beta^{-2} \sinh \beta-\beta^{-1} \cosh \beta\right), \\
& 3 A^{\prime}=\left[C^{\prime}\left(\beta^{2}+3\right)-3 D^{\prime} \beta\right] \cosh \beta-\left[3 C^{\prime} \beta-D^{\prime}\left(\beta^{2}+3\right)\right] \sinh \beta, \\
& A^{\prime} \gamma^{-2}+B_{1}^{\prime} \gamma=-\gamma .
\end{aligned}
$$

The five unknown constants appearing in the above equations can easily be solved, with the result

$$
A_{1}^{\prime}=-\Delta^{\prime} \beta \gamma^{3} R^{\prime}
$$




$$
\begin{aligned}
& B_{1}^{\prime}=3 \Delta^{\prime} \beta \gamma^{3} S^{\prime}, \\
& C^{\prime}=3 \Delta^{\prime} \beta \gamma^{3}\left[3 \alpha \cosh \alpha-\left(3+\alpha^{2}\right) \sinh a\right], \\
& D^{\prime}=3 \Delta^{\prime} \beta \gamma^{3}\left[\left(3+\alpha^{2}\right) \cosh \alpha-3 \alpha \sinh a\right], \\
& B_{3}^{\prime}=-3 \Delta^{\prime} \beta \gamma^{3},
\end{aligned}
$$

where

$$
\begin{aligned}
\Delta^{\prime}= & \left\{3\left[\beta^{2}\left(3+\alpha^{2}\right)-\alpha\left(3 \beta+\beta^{3}-\gamma^{3}\right)\right] \cosh (\beta-\alpha)\right. \\
& \left.+\left[9 \alpha \beta^{2}-\left(3+\alpha^{2}\right)\left(3 \beta+\beta^{3}-\gamma^{3}\right)\right] \sinh (\beta-\alpha)\right\}^{-1} .
\end{aligned}
$$

and $R^{\prime}$ and $S^{\prime}$ are defined by Eq. (30).

\section{References}

Bhatt, B.S., Sacheti, N.C., 1994. Flow past a porous spherical shell using the Brinkman model. Journal of Physics D: Applied Physics 27, 37-41.

Brinkman, H.C., 1947. A calculation of the viscous force exerted by a flowing fluid on a dense swarm of particles. Applied Scientfic Research A 1, 27-34.

Bungay, P.M., Brenner, H., 1973. The motion of a closely-fitting sphere in a fluid-filled tube. International Journal of Multiphase Flow 1, 25-56.

Chen, S.B., Ye, X., 2000. Boundary effect on slow motion of a composite sphere perpendicular to two parallel impermeable plates. Chemical Engineering Science 55, 2441-2453.

Debye, P., Bueche, M., 1948. Intrinsic viscosity, diffusion, and sedimentation rate of polymers in solution. Journal of Chemical Physics 16, 573-579.

Giddings, J.C., Kucera, E., Russell, C.P., Myers, M.N., 1968. Statistical theory for the equilibrium distribution of rigid molecules in inert porous networks. Exclusion chromatography. Journal of Physical Chemistry 72, 4397-4408.

Glandt, E.D., 1981. Distribution Equilibrium between a bulk phase and small pores. A.I.Ch.E. Journal $27,51-59$.

Happel, J., Brenner, H., 1983. Low Reynolds Number Hydrodynamics. Nijhoff, Dordrecht, The Netherlands.

Jones, I.P., 1973. Low Reynolds number flow past a porous spherical shell. Proceedings of the Cambridge Philosophy Society 73, 231-238.

Keh, H.J., Chiou, J.Y., 1996. Electrophoresis of a colloidal sphere in a circular cylindrical pore. A.I.Ch.E. Journal 42, $1397-1406$.

Keh, H.J., Chou, J., 2004. Creeping motion of a composite sphere in a concentric spherical cavity. Chemical Engineering Science 59, $407-415$.

Kim, S., Karrila, S.J., 1991. Microhydrodynamics: Principles and Selected Applications. Butterworth-Heinemann, Boston.

Koplik, J., Levine, H., Zee, A., 1983. Viscosity renormalization in the Brinkman equation. Physics of Fluids 26, $2864-2870$.

Makino, K., Yamamoto, S., Fujimoto, K., Kawaguchi, H., Ohshima, H., 1994. Surface structure of latex particles coverd with temperature-sensitive hydrogel layers. Journal of Colloid and Interface Science 166, 251-258.

Masliyah, J.H., Polikar, M., 1980. Terminal velocity of porous spheres. Canadian Journal of Chemical Engineers 58, $299-302$.

Masliyah, J.H., Neale, G., Malysa, K., van de Ven, T.G.M., 1987. Creeping flow over a composite sphere: Solid core with porous shell. Chemical Engineering Science 42, 245-253.

Matsumoto, K., Suganuma, A., 1977. Settling velocity of a permeable model floc. Chemical Engineering Science 32, $445-447$.

Neale, G., Epstein, N., Nader, W., 1973. Creeping flow relative to permeable spheres. Chemical Engineering Science 28, $1865-1874$.

Ooms, G., Mijnlieff, P.F., Beckers, H.L., 1970. Frictional force exerted by a flowing fluid on a permeable particle, with particular reference to polymer coils. Journal of Chemical Physics 53, 4123-4130.

Qin, Y., Kaloni, P.N., 1993. Creeping flow past a porous spherical shell. Zeitschrift fur Angewandte Mathematik und Mechanik 73, $77-84$.

Stokes, G.G., 1851. On the effect of the internal friction of fluid on pendulums. Transactions of the Cambridge Philosophy Society 9 , 8-106.

Sutherland, D.N., Tan, C.T., 1970. Sedimentation of a porous sphere. Chemical Engineering Science 25, 1948-1950.

Zydney, A.L., 1995. Boundary effects on the electrophoretic motion of a charged particle in a spherical cavity. Journal of Colloid and Interface Science 169, 476-485. 